\title{
Clinical, imaging and genetic analysis of double bilateral radix entomolaris
}

\author{
C.A. Rodríguez-Niklitschek1 , G.H. Oporto V.2, 3, I. Garay³ , L.A. Salazar² \\ ${ }^{1}$ Escuela de Odontología, Universidad Mayor, Temuco, Chile \\ ${ }^{2}$ Centro de Biología Molecular y Farmacogenética, Facultad de Medicina, Universidad de La Frontera, Temuco, Chile \\ ${ }^{3}$ Departamento de Odontología Integral Adultos, Facultad de Odontología, Universidad de La Frontera, Temuco, Chile \\ [Received 24 April 2014; Accepted 20 June 2014]
}

Background: Anatomy describes that first mandibular molars have two roots: 1 mesial, with 2 root canals, and 1 distal, with 1 root canal. The presence of three roots in these teeth is uncommon. Root anatomical variations have an impact, especially in endodontic, where the highest rates of nonsurgical treatment failures are due to the inability to identify and access roots and/or accessory canals. The aim of this research is to report a case of double three-rooted mandibular first molar through clinical, imaging and genetic analysis.

Materials and methods: Using a panoramic radiography, the presence of three roots in teeth 36 and 46 was diagnosed in a female patient. Additionally, it was indicated a cone beam computed tomography. Moreover, leukocyte genomic DNA was obtained from a blood sample of the patient to determine her ethnicity through analysis of mitochondrial DNA haplogroups using polymerase chain reaction-length restriction fragment polymorphism (PCR-RFLP).

Results: Both molars had three roots, 1 mesial (M), 1 distolingual (DL), also known as radix entomolaris (RE), and a distovestibular (DV). For both teeth, $M$ root had 2 canals, and DV and DL roots presented just 1 canal. Mitochondrial DNA analysis determined presence of haplogroup C, corresponding to Amerindian ethnicity. Conclusions: The presence of RE is uncommon. This case report contributes to describe this rare anatomical variation. To our knowledge, this is the first molecular-genetic study applied to dental anatomy and gives basis to develop future research in the area. (Folia Morphol 2015; 74, 1: 127-132)

Key words: anatomical variation, continental population groups, mitochondrial DNA, tooth root

\section{INTRODUCTION}

Current knowledge about root canals anatomy is available to clinicians. It is mainly based on researches and case reports [3]. Thus, there are several dental anatomical variations unknown [6]. The importance of understanding and achieving an accurate description of both, normal anatomy and anatomi- cal variations in teeth roots and root canal system (RCS), is relevant when dentists are going to execute procedures in zones surrounding roots and/or inside RCS. Then, exposed topics are of major interest to endodontists, considering that the main goal of endodontic treatment is to achieve a mechanical and chemical cleaning of the pulp chamber and RCS

Address for correspondence: G.H. Oporto V., DDS, MSc, PhD, Assistant Professor, Departamento de Odontología Integral Adultos, Facultad de Odontología, Universidad de La Frontera, Manuel Montt 112 Temuco, Chile, tel: 5645 2325775, e-mail: gonzalo.oporto@ufrontera.cl 
$[3,5,6,9,10]$. Within main causes of endodontic treatment failure, has been described incomplete removing of pulp tissue and microorganisms from pulp chamber and RCS [1, 3]. The causes could be a poor endodontic instrumentation technique or failure in identification of one or more root canals $[2,3,5$, $9,10]$. Thus, understanding root anatomy will contribute to improve endodontic treatment outcomes and increase success rates [2].

Mandibular first molar is one of the most treated teeth. Also, frequently needs endodontic treatment [5]. Most of anatomical researches describes that mandibular first molars have 2 roots; a mesial root, with 2 canals, and a distal root, with 1 canal $[1,6]$. However, anatomical configuration of roots and RCS of molars is variable [3]. Despite, there is not enough information available about morphological variations of RCS in specific to this tooth $[9,10]$. A possible variation is the presence of a three-rooted first molar $[1,6]$. First time described by Carabelli (1884) [cited by 1], was later denominated radix entomolaris (RE) $[1,2,5,6]$. $\mathrm{RE}$ is a supernumerary distolingual root (DL), located lingual respect to the distal root [19]. Less frequently, RE can be located in distovestibular (DV) position. When this third root is in mesiovestibular (MV) position is called radix paramolaris $[2,5]$. Usually, RE is smaller than the DV root (although could conserve a normal length) and often is curved $[1,5]$, to vestibulolingual (VL) [2]. Most cases have a severe curvature (greater than $20^{\circ}$ ), which may even get to $90^{\circ}$ at apical third $[2,6]$. RE can be separated or partially fused to other roots, and $100 \%$ of cases have a single canal, conical $[2,6]$. RE has no significant differences in prevalence by sex or by side of occurrence. Actually, in most reports, the incidence is bilateral and symmetrical [6] except in German population $[9,10]$.

To determine the presence of this root is crucial in practice of periodontology, dental surgery and is important to achieve a successful endodontic treatment $[9,10]$. RE identification requires making several radiographs at different angles. It is highly recommended taking 3 images: one image in orthoradial position, other with mesial distortion of $20-25^{\circ}$ and a third with distal distortion of $20-25^{\circ}[6,18]$. Even though retroalveolar radiography allows to identify the presence of RE, cone beam computed tomography (CBCT) provides more accurate information about inclination and curvature of RE [1] and provides a useful image for identification of the RCS and its surrounding structures.
Causal factors for this anatomical variation have not been clearly established. However, it has been hypothesized that the presence of RE could increase stability of the tooth, and would increase its alveolar bone anchorage. This additional retention might be needed in primitive human cultures that required greater masticatory efficiency. Therefore, the conservation of this third root could be a racial trait genetically determined instead of a tooth developmental abnormality $[3,11]$. Different authors propose that RE should not be considered an abnormality, rather a genetic trait [19]. In fact, researches report variability in the frequency of occurrence of RE by geographic region $[1,3,4,6,19]$. Besides, RE has a high level of genetic penetrance, and this dominance would be seen in Inuits people; Inuits "pure" and Inuits-Caucasians have a similar prevalence of this trait [6]. Also, reports indicate that exist RCS variations by ethnic origin of individuals, and these variations seems to be genetically determined $[3,19]$. Besides, studying tooth roots and root canal morphology is important not just to dentistry, also are interesting for anthropological analysis and would have a significant role as a genetic marker [19].

Chilean people have a different ethnic characterisation compared to people native form other regions worldwide. Chile has a higher frequency of Amerindian people, which reaches about $80 \%[13$, 16]. This conclusion came through the study of mitochondrial DNA markers (mtDNA). This method is based on the identification of four Native Americans haplogroups ( $A, B, C$ and $D)$ through recognition of polymorphic sites. This methodology provides unbiased information about ethnicity of people, compared to family history or the place where people live (which could lead into failures in ethnicity determinations, therefore, to incorrect associations). Determining anatomical traits based on ethnicity is important, especially to discern if those traits corresponds to normal anatomy or are, in fact, anatomical variations. Considering that researches report some physiological variants and/or pathologies are more frequently found in determined people, and otherwise, relatively uncommon in a distinct cohort $[7,8,12]$, we can state the need to investigate anatomical variations of teeth and its characteristics in different ethnic groups.

This study analyse under morphological, imagenological and genetic perspectives, a case of double $\mathrm{RE}$ in mandibular first molars. 

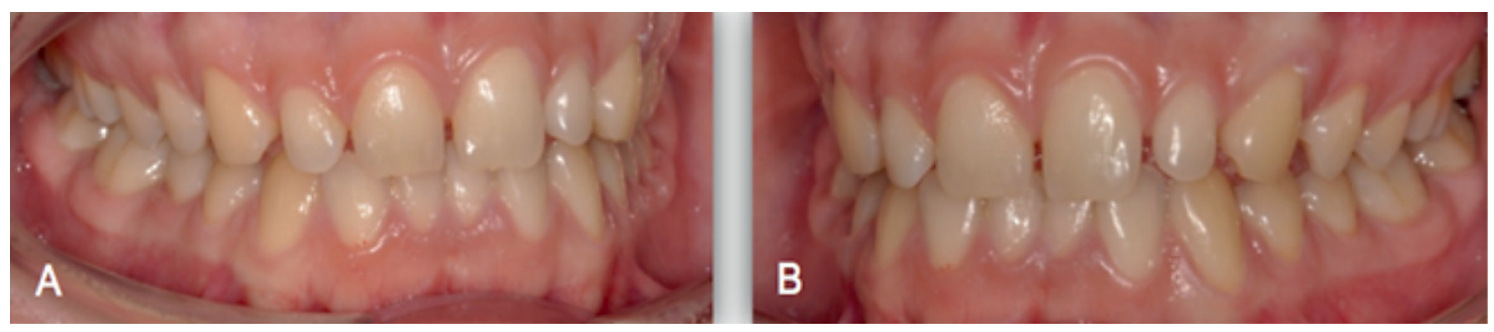

Figure 1. Front-right view (A) and front-left view (B) of maxilla and mandible.

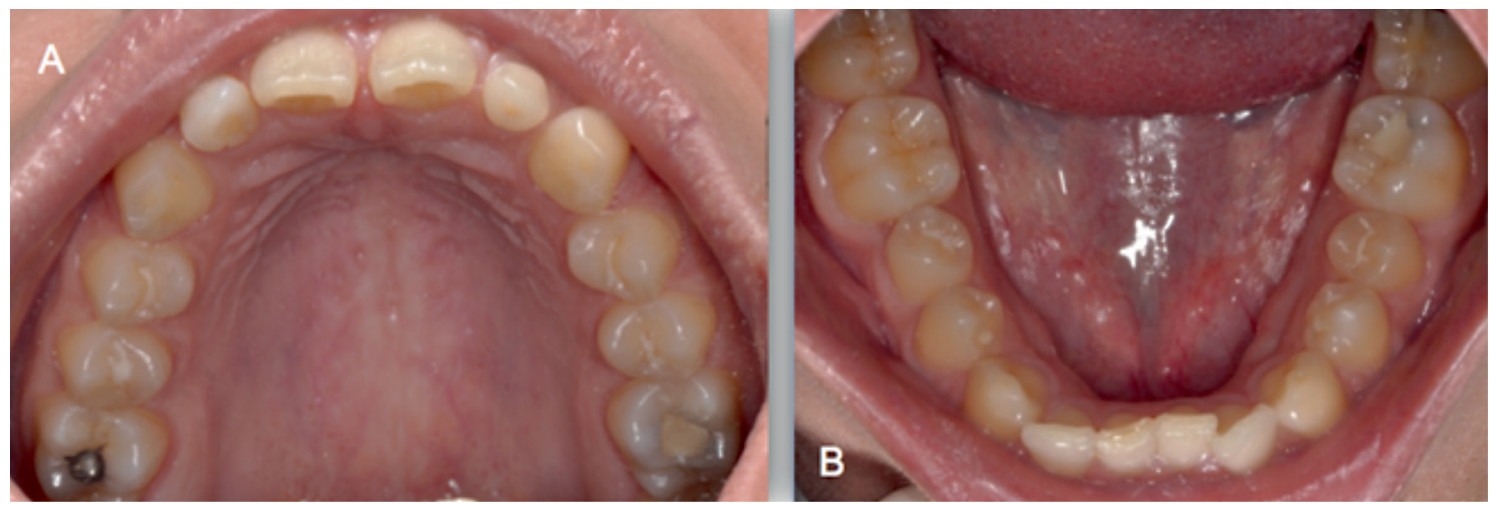

Figure 2. Maxillary occlusal view (A) and mandibular occlusal view (B).
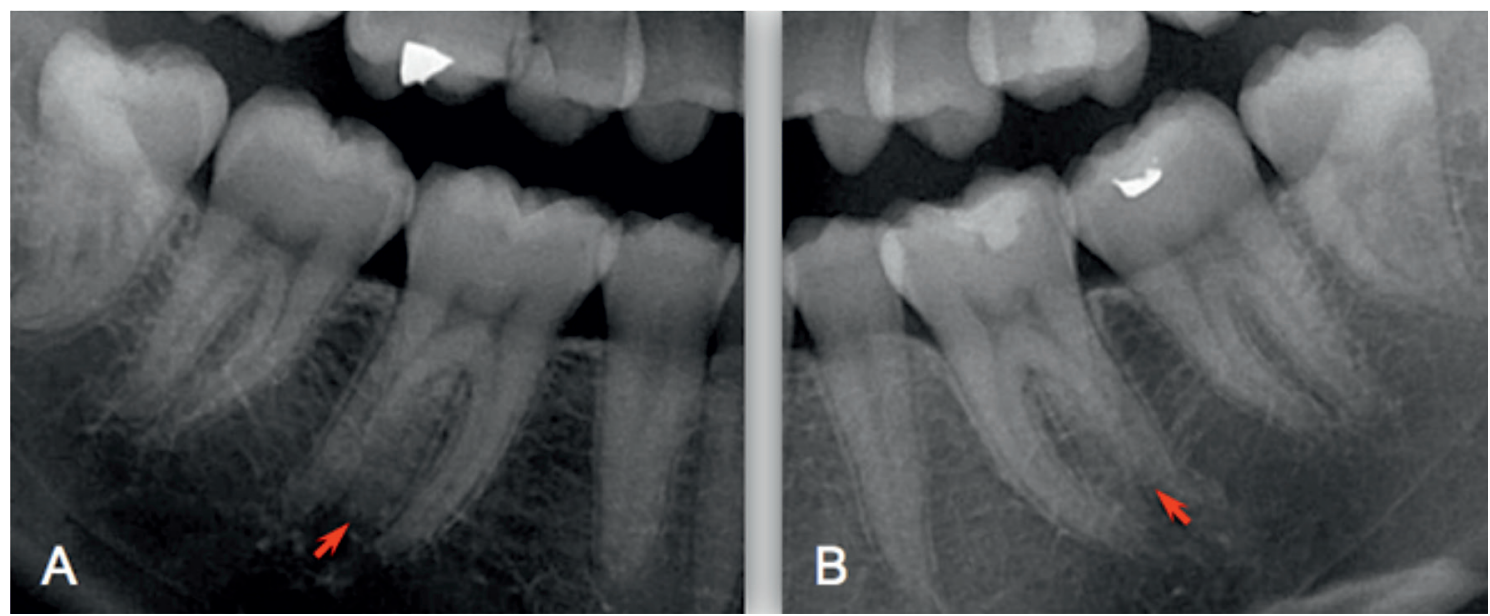

Figure 3. Detailed analysis of panoramic radiograph; tooth 46 (A) and 36 (B). Notice presence of both teeth three-rooted.

\section{CASE REPORT}

A female patient (27 years old) went to the dentist office for a preventive dental examination. During anamnesis, patient did not report any systemic or oral diseases. At clinical examination, it was not observed caries or other pathology (Fig. 1). Some teeth had restorations with some aesthetic requirements (Fig. 2). To complement clinical examination, we indicated a panoramic radiograph and bilateral bitewing radiographs. These exams did not show any pathology. It was indicated a scaling and a prophylaxis as preventive treatment.

During analysis of panoramic radiograph, the presence of three roots at teeth 36 and 46 was suspected (Fig. 3). Due to the unusual of the case, and with the aim of discard the presence of other pathology 


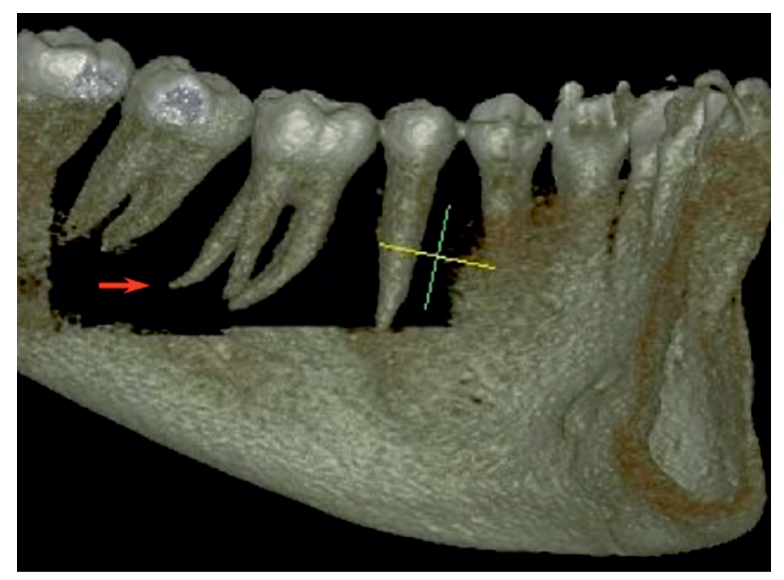

Figure 4. Cone beam computed tomography lingual view of tooth 36 ; radix entomolaris arrowed.

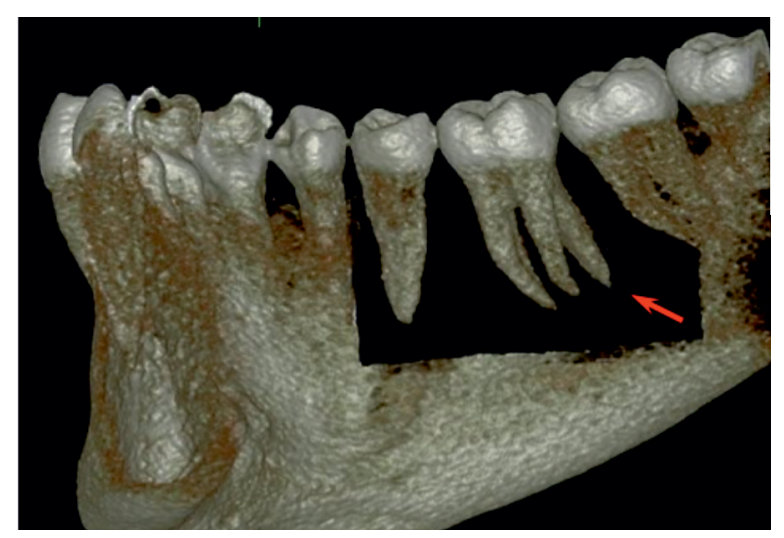

Figure 5. Cone beam computed tomography lingual view of tooth 46 ; radix entomolaris arrowed. (especially neoplastic) a CBCT was indicated. Patient was immediately notified about the situation, and she affirmed that never before was informed about it. Patient signed an informed consent form, and then a $C B C T$ was made to establish the shape, number and orientation of the roots of the teeth 36 and 46 . Also, a blood sample was taken. Leukocyte genomic DNA was extracted to confirm the ethnicity of the patient through mtDNA haplogroups analysis. This procedure was performed using polymerase chain reaction-length restriction fragment polymorphism (PCR-RFLP) methodology previously described by Lagos et al. [12].

Subsequent to an accurate analysis of images obtained with $C B C T$, we determined that both first mandibular molars had three roots: mesial root, DL root (RE) and DV root (Figs. 4, 5). To both teeth, mesial root had two canals, and DV; besides DL roots displayed only one canal (Figs. 6, 7). There was not found any pathology associated. Mitochondrial DNA analysis demonstrated the presence of $\mathrm{C}$ haplogroup, corresponding to Amerindian ethnicity of patient (Fig. 8).

\section{DISCUSSION}

Human teeth are not invariables structures, so, are able to have morphological alterations. For each type of teeth, is possible to observe anatomical variations with variable frequencies. The presence of three-rooted mandibular molars is uncommon. This case report describes a bilateral first mandibular molar RE, considered an unknown anatomical variation [4].

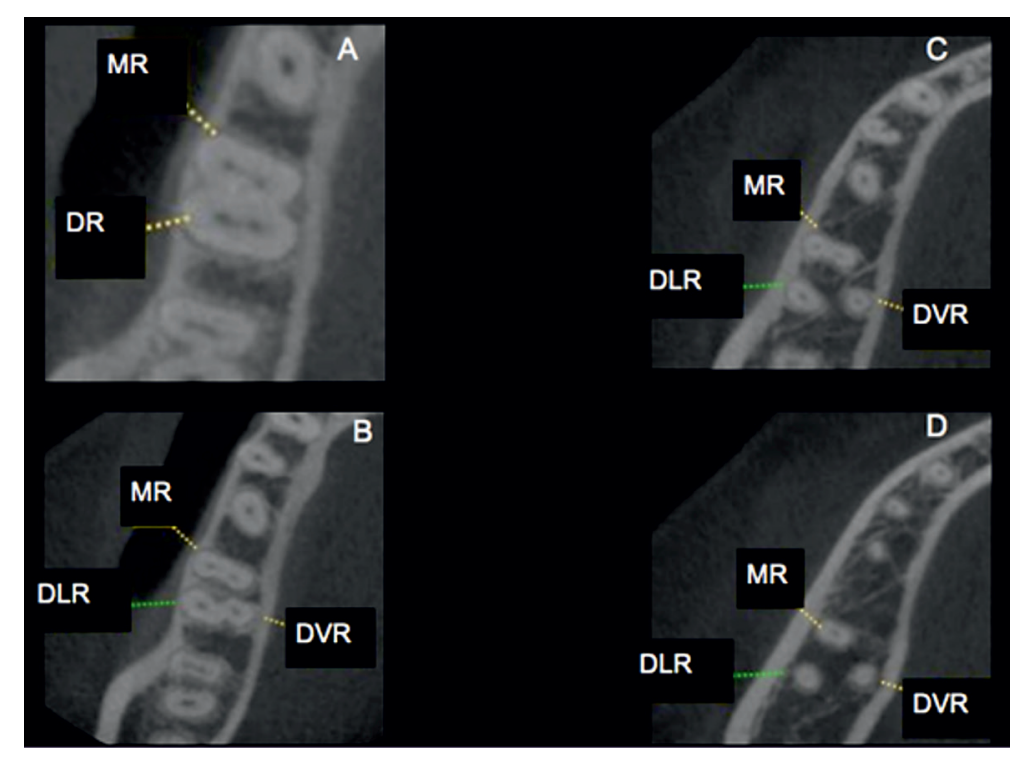

Figure 6. Cone beam computed tomography cross section of tooth 36; cervical (A), medium (B, C) and apical (D); MR — mesial root; DR distal root; DLR — distolingual root; DVR — distovestibular root. 


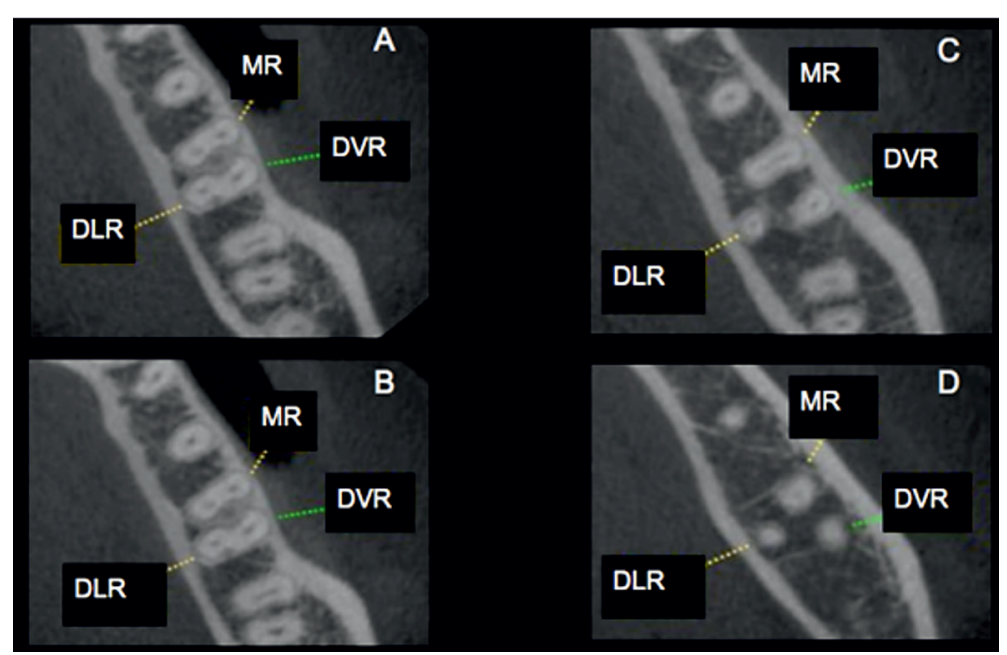

Figure 7. Cone beam computed tomography cross section of tooth 46; cervical (A), medium (B, C) and apical (D); MR - mesial root; DLR distolingual root; DVR — distovestibular root.

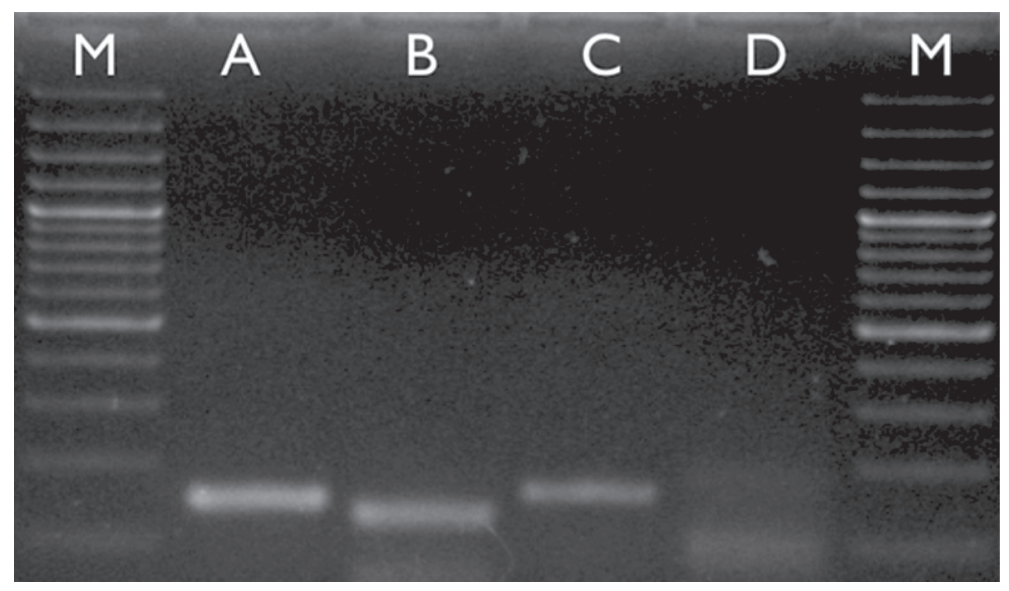

Figure 8. Agarose gel $2 \%$ electrophoresis showing results of polymerase chain reaction-length restriction fragment polymorphism (PCR-RFLP) mtDNA Amerindian haplogroups according to methodology described by Lagos et al. [12]. Lines A, B, C and D showed Amerindians haplogroups $A, B, C$ and D, respectively. Molecular-weight size marker at line $M$. Line $C$ shows loss of a restriction site to Hincll enzyme indicating patient had Amerindian haplogroup $\mathrm{C}$.

In dentistry, clinical importance of three-rooted mandibular first molars are mainly in procedures to be performed close and/or in structures that are surrounding teeth roots (periodontology and oral surgery) and within the RCS. Here rises the importance of RE in endodontic treatments, where the highest rates of non-surgical treatment failures are due to the inability to identify and access roots and/or accessory canals. Therefore, is necessary to proceed carefully during treatment, and analyse radiographs in order to discover a possible supernumerary root. Determining presence of RE in mandibular first molars may be of major importance to achieve a successfully endodontic treatment, especially in complex cases, where clinicians must anticipate, assume their existence and look for an additional root.

Root anatomical variations are not always associated to a variation in the tooth crown that suggests an altered root form [15]. Therefore, in dental practice, a detailed radiographic analysis is necessary, especially prior to performing procedures within the RCS $[14,15,17]$. Existing results are consistent with this statement, where size and volume of molars crowns did not lead to presume any alteration in roots form (Fig. 2B).

Although literature indicates that CBCT has a lower resolution than the retroalveolar radiograph $[1,2]$, $C B C T$ provides more and better information about orientation, shape and number of tooth roots, RCS and 
surrounding structures $[1,9,10,19]$ so, CBCT would be the preferred imaging exam to study the anatomy of RCS. Results of dental imaging studies are consistent with literature reports. Analysing radiographies (Fig. 3) and comparing it to $C B C T$ images (Figs. 4-7) is clear that $\mathrm{CBCT}$ delivers more information about shape, number and orientation of roots and root canals.

Available data indicates that within the most probable causes of RE are genetic factors. Interestingly, there are not genetic or molecular studies supporting this theory. Actually, it is based only on RE frequency observed in specific ethnic groups, and ethnicity was determined exclusively based on geographical residence and/or using family names of people studied $[2,3,5$, $6,9,10,19]$. Determining ethnic backgrounds using these strategies may deliver incorrect data, because ethnic composition of people is heterogeneous due to migration. Adding that RE is an anatomical variation that has been reported in different populations worldwide, it raises the need to determine ethnicity through objective tools. Thus, nowadays these determinations should be done through molecular biology techniques that deliver objective data, helping to characterise a population more accurately. An available method is mtDNA haplogroups analysis [12]. This methodology assigns a precise ethnic characterisation. Then, we can correlate anatomical (and/or physiologic) traits to an ethnic origin and, finally, conclude whether a structure (or function) is normal or is a variation in specific people $[7,8,12]$. Consulted literature indicates that this would be the first case report linking a dental morphological structure (considered an unusual anatomical variation) with molecular determination of ethnicity. Although results are not enough to indicate that RE is an Amerindian trait, provides background for further studies, which will establish dental morphological characteristics of people according to ethnicity (determined through molecular biology). Besides the importance of this topic in clinical dentistry, describing and understanding normal dental structures and those considered anatomic variations by ethnic origin are relevant as well from anthropological sciences perspective [19].

Finally, determining dental anatomical features for each ethnic group will lead to improving prognosis of treatments and will minimise accidents during RCS procedures.

\section{REFERENCES}

1. Abella F, Mercadé $M$, Duran-Sindreu F, Roig M (2011) Managing severe curvature of radix entomolaris: three-dimensional analysis with cone beam computed tomography. Int Endod J, 44: 876-885.
2. Abella $F$, Patel $S$, Durán-Sindreu $F$, Mercadé $M$, Roig $M$ (2012) Mandibular first molars with disto-lingual roots: review and clinical management. Int Endod J, 45: 963-978.

3. Al-Qudah A, Awawdeh L (2009) Root and canal morphology of mandibular first and second molar teeth in a Jordanian population. Int Endod J, 42: 775-784.

4. Calberson FL, De Moor RJ, Deroose CA (2007) The radix entomolaris and paramolaris: clinical approach in endodontics. J Endod, 33: 58-63.

5. Chandra SS, Chandra S, Shankar P, Indira R (2011) Prevalence of radix entomolaris in mandibular permanent first molars: a study in a South Indian population. Oral Surg Oral Med Oral Pathol Oral Radiol Endod, 112: 77-82.

6. De Moor RJ, Deroose CA, Calberson FL (2004) The radix entomolaris in mandibular first molars: an endodontic challenge. Int Endod J, 37: 789-799.

7. Guzman N, Lanas F, Salazar L (2010) Influence of Amerindian mitochondrial DNA haplogroups on thrombosis susceptibility and frequency of four genetic prothrombotic variants in southern Chilean subjects. Clin Chim Act, 411: 444-447.

8. Guzman N, Salazar L (2010) Frecuency of prothrombotic risk factors in patients with deep venous thrombosis and controls: Their implications for thrombophilia screening in Chilean subjects. Genet Test Mol Biomark, 14: 1-4.

9. Huang C, Chang Y, Chuang M, Lai T, Lai J, Lee B, Lin C (2010) Evaluation of root and canal systems of mandibular first molars in Taiwanese individuals using cone-beam computed tomography. J Formos Med Assoc, 109: 303-308.

10. Huang R, Cheng W, Chen C, Lin C, Lai T, Shen E, Chiang C, Chiu H, Fu E (2010) Three-dimensional analysis of the root morphology of mandibular first molars with distolingual roots. Int Endod J, 43: 478-484.

11. Kupczik K, Hublin J (2010) Mandibular molar root morphology in Neanderthals and Late Pleistocene and recent Homo sapiens. J Hum Evol, 59: 525-41.

12. Lagos J, Lemus J, Sierra F, Mella R, Fuentes F, Ocares G, Rosales A, Salazar L, Durán D, Guzmán N (2010) Asociación de niveles de lípidos y haplogrupos Amerindios de DNA mitocondrial en individuos chilenos hipercolesterolémicos tratados con Atorvastatina. Rev Chil Cardiol, 29: 208-213.

13. Moraga ML, Rocco P, Miquel JF, Nervi F, Llop E, Chakraborty R, Rothhammer F, Carvallo P (2000) Mitochondrial DNA polymorphisms in Chilean aboriginal populations: implications for the peopling of the southern cone of the continent. Am J Phys Anthropol, 113: 19-29.

14. Oporto VGH, Fuentes FRE, Soto PCC (2010) Tooth root and pulp canal anatomical variations. Int J Morphol, 28: 945-950.

15. Oporto VGH, Saavedra R, Fuentes R, Soto PCC (2013) Double root anatomical variations in a single patient: endodontic treatment and rehabilitation of a three-rooted first premolar. Case report. Int J Morphol, 31: 45-49.

16. Rocco P, Morales C, Moraga M, Miquel JF, Nervi F, Llop E, Carvallo P, Rothhammer F (2002) Genetic composition of the Chilean population. Analysis of mitochondrial DNA polymorphisms. Rev Méd Chile, 130: 125-131.

17. Soares J, Leonardo R (2003) Root canal treatment of threerooted maxillary first and second premolars: a case report. Int Endod J, 36: 705-710.

18. Wang Q, Yu G, Zhou XD, Peters OA, Zheng QH, Huang DM (2011) Evaluation of $x$-ray projection angulation for successful radix entomolaris diagnosis in mandibular first molars in vitro. J Endod, 37: 1063-1068.

19. Zhang R, Wang H, Tian $Y, Y u X$, Hu T, Dummer $P$ (2011) Use of cone-beam computed tomography to evaluate root canal and canal morphology of mandibular molars in Chinese individuals. Int Endod J, 44: 990-999. 